\title{
Evolution of BRET biosensors from live cell to tissue-scale in vivo imaging
}

\section{Abhijit De ${ }^{1}{ }^{*}$, Akshi Jasani ${ }^{1}$, Rohit Arora ${ }^{1}$ and Sanjiv S. Gambhir ${ }^{2}$}

${ }^{1}$ Molecular Functional Imaging Laboratory, ACTREC, Tata Memorial Centre, Navi Mumbai, India

${ }^{2}$ MIPS, Department of Radiology, School of Medicine, Stanford University, Stanford, CA, USA

\section{Edited by:}

Milka Vrecl, University of Ljubljana,

Slovenia

Reviewed by:

Brian Hudson, University of Glasgow, UK

Stuart Maudsley, National Institutes of Health, USA

${ }^{*}$ Correspondence:

Abhijit De, Molecular Functional

Imaging Laboratory, ACTREC, Tata

Memorial Centre, Sector 22.

Kharghar, Navi Mumbai 410210, India

e-mail: ade@actrec.gov.in
Development of bioluminescence resonance energy transfer (BRET) based genetic sensors for sensing biological functions such as protein-protein interactions (PPIs) in vivo has a special value in measuring such dynamic events at their native environment. Since its inception in the late nineties, BRET related research has gained significant momentum in terms of adding versatility to the assay format and wider applicability where it has been suitably used. Beyond the scope of quantitative measurement of PPIs and protein dimerization, molecular imaging applications based on BRET assays have broadened its scope for screening pharmacologically important compounds by in vivo imaging as well. In this mini-review we focus on an in-depth analysis of engineered BRET systems developed and their successful application to cell-based assays as well as in vivo non-invasive imaging in live subjects.

Keywords: bioluminescence resonance energy transfer, luciferase, fluorescent proteins, optical imaging, protein-protein interactions, cell-based assay

\section{INTRODUCTION}

In the post-genomic era, rapid functional evaluation of proteinprotein interactions (PPIs), protein phosphorylation, or protease function, which play a key role in various cellular processes such as signal transduction, cell division, transport, etc., in live cell condition is essential. Moreover, the study of such PPIs in normal and diseased cells can help shed light in the understanding of the diseases and to develop suitable therapies. For a long time, conventional biochemical assays like co-immunoprecipitation (1, 2), gel-filtration chromatography (3), sandwich enzyme-linked immunosorbent assay (ELISA) (4), etc., have been used in the investigation of PPIs. These assays though successful, do not suffice as imaging probes because they: (i) are essentially endpoint measurements, (ii) fail to provide spatio-temporal information on specific PPIs, (iii) require mechanical, chaotropic, or detergent based cell lyses, which may alter native PPIs in some cases $(5,6)$, (iv) are insensitive to transient interactions that regulate certain cellular processes, and (v) have little or no utility for in vivo imaging in live subjects. To overcome these limitations, non-invasive imaging approaches such as bioluminescence resonance energy transfer (BRET) have been developed over the last decade, which allow the study of PPIs in their native environment and are capable of providing a unified platform that can be translated from cell culture-based assays to the imaging of live subjects $(6,7)$. In this mini-review, we will be exploring some hitherto unexplained factors affecting the spectral pattern of several BRET systems and their successful application to cell-based assays as well as in vivo imaging of live subjects.

\section{BIOPHYSICAL BASIS OF BRET}

Bioluminescence resonance energy transfer is an intrinsic phenomena occurring in the organisms Renilla reniformis and
Aequorea victoria. Exploiting the underlying principles of BRET from nature, literatures demonstrating BRET biosensor applications started since the year 1999. The BRET phenomenon that follows the Förster resonance energy transfer (RET) principle (8), occurs between two proximally situated chromophores - a bioluminescent donor such as a luciferase protein and a fluorescent protein (FP) acceptor with overlapping emission and excitation spectra respectively. Following donor excitation upon substrate addition, part of the electronic excitation energy of the donor is dissipated due to random collisions with other molecules while the remaining electronic relaxation energy is transferred to the acceptor molecule through non-radiative dipole-dipole coupling. Upon excitation, the acceptor molecule now emits its photonic energy at its characteristic wavelength. This results in a decrease in donor emission paralleled by an increase in acceptor emission. The strict dependence of BRET on the inter-chromophoric distance $(1-10 \mathrm{~nm})$ makes it an appropriate "molecular yardstick" for determining PPIs. This is true, since the average protein radius is $\sim 5 \mathrm{~nm}$, which means that a positive BRET signal will only be detected if the two proteins come within $\sim 10 \mathrm{~nm}$ of each other, a distance that is an indicator of direct interaction between the two proteins (9). However, absence of a BRET signal does not necessarily mean that the two target proteins do not interact with each other. Lack of a signal can be accounted for by an unfavorable orientation between the donor and acceptor dipoles. The BRET ratios can be calculated as per Eqs. 1 and 2 (10).

$\operatorname{BRET}=\frac{\mathrm{BL}_{\text {emission }}(\text { Acceptor } \lambda)-C_{\mathrm{f}} \times \mathrm{BL}_{\text {emission }}(\text { Donor } \lambda)}{\mathrm{BL}_{\text {emission }}(\text { Donor } \lambda)}$ 
where,

$$
C_{\mathrm{f}}=\frac{\mathrm{BL}_{\text {emission }}(\text { Acceptor } \lambda)_{\text {donor only }}}{\mathrm{BL}_{\text {emission }}(\text { Donor } \lambda)_{\text {donor only }}}
$$

In the above equation, $\mathrm{BL}_{\mathrm{emission}}$ is the average radiance measured at the donor (Donor $\lambda$ ) or acceptor (Acceptor $\lambda$ ) filters in BRET-transfected or only donor transfected cells; the correction factor $\left(C_{\mathrm{f}}\right)$ represents the BRET signal detected from cells transfected only with the donor plasmid. Upon subtracting this factor from the overall BRET ratio, one can get an idea of the dynamic range for a particular BRET pair. Moreover, since BRET-based assays are ratiometric, any variability due to assay volume or cell number variation or time point of measurement is nullified.

Until recently, the field of BRET-based biosensors has predominantly utilized two basic BRET systems, viz., BRET ${ }^{1}$ and $\mathrm{BRET}^{2}$. Developed by Xu et al. the BRET ${ }^{1}$ system combines Renilla luciferase (RLuc) with enhanced yellow fluorescent protein (EYFP) (11). However, the spectral resolution (separation of peak donor and acceptor emission spectra) achieved in BRET ${ }^{1}$ is $\sim 50 \mathrm{~nm}$ only, which is considered suboptimal for macroscopic imaging $(12,13)$. Another BRET system, named as BRET ${ }^{2}$, combining RLuc with a UV-excitable GFP variant viz., GFP ${ }^{2}(14,15)$ was developed, that uses a coelenterazine analog-DeepBlueC ${ }^{\mathrm{TM}}$ (also known as coelenterazine 400a or Clz400) substrate, which shifts the emission maximum $\left(E_{\mathrm{m}_{\max }}\right)$ of RLuc to $400 \mathrm{~nm}$. GFP ${ }^{2}$ excites at a maximum $\left(E_{\mathrm{x}_{\max }}\right)$ of $396 \mathrm{~nm}$ and emits photons at $510 \mathrm{~nm}$. This yields a much larger spectral resolution of $110 \mathrm{~nm}$ and has enabled us to perform tissue-scale imaging using wideband filters for the first time $(16,17)$. However, successful tissue imaging with higher sensitivity of cells located deep inside the animal body calls for the design and development of BRET systems with more red-shifted emissions. This is because, at wavelengths below $600 \mathrm{~nm}$, particularly in the blue-green regions of light, pigments like myoglobin and hemoglobin absorb a significant fraction of the visible light (18).

\section{EXPANSION OF BRET ASSAY FORMATS}

In the past few years, improvisations in various components of BRET such as luciferases, FPs, substrates, and instrumentations have contributed to the remarkable expansion in the range of BRET platforms available. Armed with these BRET vectors, the progress of molecular imaging to live cells, animals, and plants with varied applications has been made possible. With the advent of engineered RLuc variants with an elevated photon output and/or a red-shifted $E_{\mathrm{m}_{\max }}$, viz., RLuc8 $\left(E_{\mathrm{m}_{\max }} 480 \mathrm{~nm}\right.$; four fold increase in photon output compared to RLuc) (19) and RLuc8.6 $\left(E_{\mathrm{m}_{\max }} 535 \mathrm{~nm} ; \sim 6\right.$-fold increase in photon output compared to RLuc) (20), new BRET systems in combination with FPs in the orange and red regions of emission spectra were developed (Figures 1A-D). Theoretically, the amplitude of donor emission should always exceed the acceptor emission (Figures 1A,C). However, we noted that in the spectral profiles of some of these newly developed BRET systems, the normalized amplitude at the donor emission was lower in comparison to that at the acceptor emission (Figure 1B). For example, in the case of TagRFP-RLuc8, only when Clz- $v$ substrate was used (shifting the peak donor emission to $515 \mathrm{~nm}$ ), the amplitude of TagRFP at $585 \mathrm{~nm}$ surpassed
RLuc8 emission. To explain this anomaly, a deeper understanding of the RET principle is required. RET efficiency is essentially an interplay between the spectral overlap integral of the donor emission and acceptor excitation spectra, in addition to the quantum yield of the donor. We speculate that a donor bleed through signal coupled with the high degree of spectral overlap between RLuc8 and TagRFP (upon the use of Clz- $v$ ) that favors maximum energy transfer between the pair is detected at the acceptor filter, giving an unnaturally high peak. On a different note, if one tries to define the ideal BRET pair for tissue-scale imaging, it would be the one that gives a high spectral resolution with minimally compromising the BRET ratio. Based on the data compiled from the BRET systems available with us (Figure 1E), TurboFP and RLuc8.6-Clz combination would be the ideal BRET partners for both in vitro and in vivo imaging as they have a high BRET ratio $(\sim 1.19)$ with an equally high spectral separation of $100 \mathrm{~nm}$.

\section{BRET OPTIONS FOR STUDYING THE KINETICS OF PROTEIN INTERACTIONS}

A landmark development in the recruitment of BRET-based systems to capture the PPI kinetics was the creation of protected $\mathrm{Clz}$ analogs. The problem associated with the use of normal Clz substrates in live cells was their auto-oxidation, resulting in the loss of peak signal within $2-3 \mathrm{~min}$, which further drops to $\sim 50 \%$ within $17 \mathrm{~min}$. Consequently the measurement of long-term PPI kinetics is impossible using such substrates. To eliminate this problem, Levi et al. (21) reported chemical modifications to protect the putative oxygenation sites of $\mathrm{Clz} 400$ and demonstrated that depending on the protective modifications, long-term BRET $^{2}$ monitoring was achievable. Similarly, another commercial source also developed EnduRen $^{\mathrm{TM}}$ substrate that can be used specifically for live cell imaging (22). This is a protected form of coelenterazine- $h$ with their active sites blocked by esters or oxymethyl ethers that are only released upon cleavage by intracellular hydrolytic enzymes. The absence of active Clz- $h$ in the media significantly reduces the signal attenuation due to background auto-oxidation and the half-life of Clz- $h$ increases. Moreover, a steady-state bioluminescence emission of EnduRen ${ }^{\mathrm{TM}}$ till about $24 \mathrm{~h}$ potentiates its use for monitoring dynamic changes in PPIs from live cell conditions.

Another commonly used luciferase is the North American Firefly luciferase (FLuc; $E_{\mathrm{m}_{\max }} 562 \mathrm{~nm}$ ) (23). A codon-optimized version of FLuc has been created by commercial sources for use in mammalian cells. The relatively slower and stable emission kinetics of its substrate, D-luciferin, makes it naturally suitable for kinetic measurements from live environments, obviating the need for any chemical modifications to its structure (24). FLuc has been reported to be used in a BRET system in conjunction with red FPs like DsRed (25) as well as with non-protein fluorophores such as Cy3 and Cy3.5 (26). However, its bulky size of $61 \mathrm{kDa}$, an obligate dependence on $\mathrm{Mg}^{2+}$ and ATP as its cofactors $(27,28)$ and finally, a low spectral resolution with the BRET partners reported so far, makes it a poor choice for BRET.

\section{MULTIPLEXED BRET OPTIONS FOR CO-LATERAL INTERACTION STUDIES}

In addition to the simple PPIs assays, one might be interested to monitor two concurrent dependent/independent PPI events 


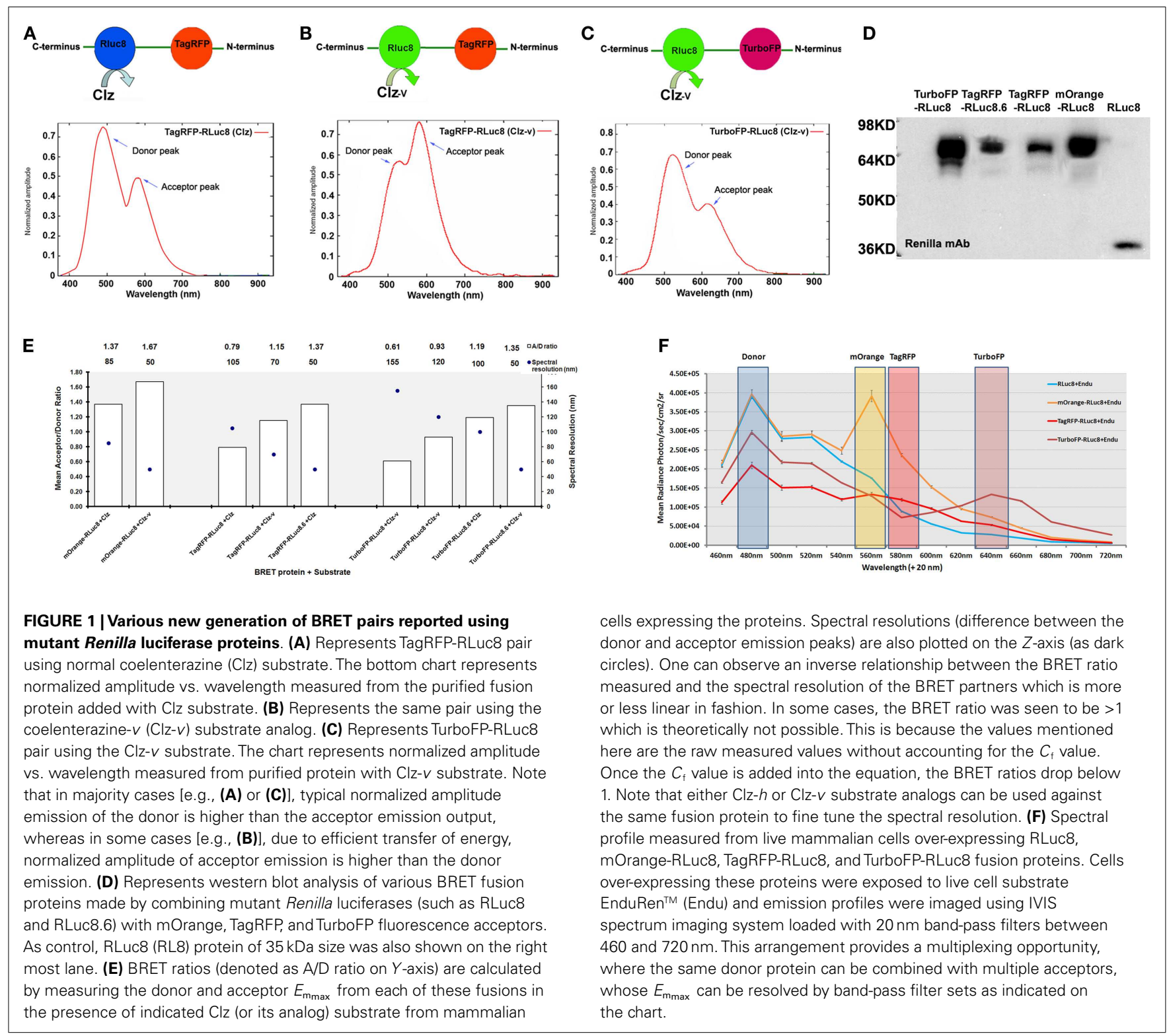

within the same cell. BRET multiplexing was employed in one of the GPCR studies to monitor the ubiquitination kinetics and its involvement in receptor regulation. Exploiting the distinct spectral emission properties of the RLuc substrates-Clz- $h\left(E_{\mathrm{m}_{\max }} 480 \mathrm{~nm}\right)$ and Clz400 ( $\left.E_{\mathrm{m}_{\max }} 400 \mathrm{~nm}\right)$, Perroy et al. co-expressed RLuc- $\beta$ arrestin and $\mathrm{GFP}^{2}$-ubiquitin along with a YFP-labeled vasopressin receptor $\left(\mathrm{V}_{2} \mathrm{R}\right.$-YFP) (29). In this way, depending on the substrate (Clz- $h$ or Clz400) oxidized by RLuc, either BRET ${ }^{1}$ or BRET ${ }^{2}$ kinetics can be respectively detected. Appropriate negative controls, for instance, the use of Clz- $h$ to detect negligible BRET transfer between RLuc and GFP ${ }^{2}$ can validate the authenticity of such experiments. Moreover, with the series of BRET systems that are now available to us, one can recruit either a single/dual luciferase system such as RLuc8 and RLuc8.6 with appropriate acceptor FPs (Figure 1F), which can facilitate BRET multiplexing of three to four candidate proteins. The ease with which this objective can be achieved and the requirement of only a single substrate, makes it a highly attractive option for co-lateral protein interaction studies.

\section{MULTIPLEXED BRET OPTIONS FOR STUDYING MULTI-PROTEIN COMPLEX}

While dual-BRET techniques facilitate the concomitant monitoring of two different PPIs events, elegant approaches such as sequential RET (SRET) (30, 31), bimolecular-fluorescence complementation-BRET (BiFC-BRET) $(32,33)$, complemented donor-acceptor-RET (CODA-RET) (34), and bimolecular luminescence complementation-BiFC (BiLC-BiFC) $(35,36)$ have enabled the detection of interactions between higher order protein complexes. In the SRET technique, the three candidate proteins are fused to either RLuc donor or one of the two FP acceptors. In such a situation, a BRET process excites the first fluorescent acceptor, which will now serve as a fluorescence resonance energy transfer 
(FRET) donor for the second fluorescent acceptor. Two such systems, SRET $^{1}$ (RLuc-YFP-DsRed) and SRET ${ }^{2}$ (RLuc-GFP ${ }^{2}$-YFP) utilizing $\mathrm{Clz}-h$ and $\mathrm{Clz} 400$ substrates respectively, were reported in literature, that could detect the heterotrimerization of cannabinoid $C_{1}$ receptor $\left(C B_{1} R\right)$, dopamine $D_{2}$ receptor $\left(D_{2} R\right)$, and adenosine $A_{2 A}$ receptor $\left(A_{2 A} R\right)$ as well as the assembly of $G$ protein subunits in living cells. Further, Navarro et al. successfully demonstrated the oligomerization of Calmodulin ( $\mathrm{CaM}), \mathrm{A}_{2 \mathrm{~A}} \mathrm{R}$, and $\mathrm{D}_{2} \mathrm{R}$ using SRET ${ }^{2}$ in live cells, which can open avenues for screening of potential drugs that specifically target these receptor interactions. In another study, the $\mathrm{CB}_{1} \mathrm{R}-\mathrm{D}_{2} \mathrm{R}-\mathrm{A}_{2 \mathrm{~A}} \mathrm{R}$ interactions were studied using a $\mathrm{BiFC}$ (using $\mathrm{N}$ - and $\mathrm{C}$-termini truncated forms of YFP) coupled with a luciferase protein to form a functional BRET system (BiFC-BRET). In yet another recent literature, a split luciferase complementation reconstituting the donor bioluminescence was paired to an acceptor FP to detect the BRET signal. This technique, termed CODA-RET can be used in the study of receptor oligomerization in presence of agonists/antagonists as well as in drug screening. Amalgamating the above two techniques, one can also employ both complemented donor along with bimolecuar fluorescence complementation (BiLC-BiFC) to form a functional BRET system that can explore the interaction of up to four proteins.

\section{BRET FOR TISSUE-SCALE IMAGING}

While we have progressed so far in terms of optimizing various BRET platforms with the aim to image PPIs non-invasively in their natural physiological environments in vivo, it has not yet been achieved completely. However, scientific endeavors have not been futile. With the introduction of the intensely cooled charge coupled device (CCD) camera-based optical imaging instrumentation, the ability to detect very dim photon signals from live cells in culture or from animal or plant tissues has become possible. To detect signals with detectors placed outside the animal subjects, the cells of interest present at a depth within the subject must produce sufficient signal. Here, primarily the use of red and NIR light signals is favored as they have lesser tissue attenuation and thus, better penetration capacity. Therefore, overall modification of existing assays to adapt them for non-invasive monitoring is a challenging task. Approaching the development of a single format imaging assay that can serve to measure PPIs from isolated single cells as well as physiologically relevant animal/plant models, both $\mathrm{BRET}^{1}$ and $\mathrm{BRET}^{2}$ strategies display some form of confinements. Therefore, while attempting live animal BRET assays, we have conducted serial experiments to identify an optimal BRET assay showing satisfactory performance as a single format assay $(12,16,17)$. By now, we have introduced an ample variety of the red light emitting BRET vectors, many of which undoubtedly show superior performance over the previous assays used. By withdrawing the traditional method of BRET measurement using a microplate reader, we adapted a method for spectral separation of donor and acceptor signal by using black-box cooled CCD camera macro-imager (16). An important parameter to successfully adapt this imaging method was the use of the BRET formats with relatively large spectral resolution, which allows the selection of wide band-pass emission filters in the device. Thus the CCD camerabased macro-imaging instrument can measure BRET signals from lysed or live cells placed in multi-well plates. The same instrument can then be used for BRET measurement from whole organisms as well. A point worth noting here is that, BRET imaging from animal tissues is further complicated by the consideration of tissue attenuation factor. To address this, a double ratio (DR) which provides a depth-independent measure of the BRET signal in animal experiments was defined (Eq. 3) (7).

$$
\begin{aligned}
\mathrm{DR}= & \frac{\mathrm{BL}_{\text {emission }}(\text { Acceptor } \lambda)_{\mathrm{BRET}} \times \mu_{t}(\text { Acceptor } \lambda)}{\mathrm{BL}_{\mathrm{emission}}(\text { Donor } \lambda)_{\mathrm{BRET}} \times \mu_{t}(\text { Donor } \lambda)} / \\
& \frac{\mathrm{BL}_{\mathrm{emission}}(\text { Acceptor } \lambda)_{\text {donoronly }} \times \mu_{t}(\text { Acceptor } \lambda)}{\mathrm{BL}_{\mathrm{emission}}(\text { Donor } \lambda)_{\text {donoronly }} \times \mu_{t}(\text { Donor } \lambda)}
\end{aligned}
$$

where, $\mu_{t}$ denotes the total attenuation coefficient.

The main bottleneck of extending FRET strategy in small animal evaluation is associated with the auto-fluorescence correction method. As light travels in and out from animal tissues, the resulting photon attenuation complicates the FRET ratio calculations. In this context, the exclusion of an external photon input makes BRET-based technologies more acquiescent for macro-scale imaging of PPIs. As represented in Figure 2, we have also done proof of principle studies by confirming the detection of the rapamycindependent interaction of FKBP12 and FRB from living animals ( 7 , $12,17)$. Following the successful BRET imaging from small animal model, macro-imaging of plant tissues was also reported (37). Using a modified electron bombardment-CCD camera coupled with a dual-view image splitter, visualization of the constitutive photomorphogenesis 1 protein (COP1) homo-dimerization using RLuc-EYFP BRET assay was demonstrated in the rootlet and cotyledons of tobacco seedlings in order to understand its repressive activity on light regulated development in plants. The same group had previously reported the use of a similar BRET assay in onion epidermal cells as well as in the Arabidopsis seedlings to study the effect of COP1 dimerization and its nuclear exclusion on the functional activity of COP1 (38). BRET is better adapted to plant imaging, since it circumvents the issues of photobleaching and auto-fluorescence of photosynthetic pigments as seen in the case of FRET. Considering careful validation of the PPIs in systematic, large-scale models using individual test cases, the molecular imaging assays like BRET appear promising in the current proteomic developments. So far, the major hurdle with BRET strategy was our inability to visualize the interactions of endogenous proteins. However, this is no longer an impediment, as Audet et al. have successfully reported the measurement of BRET signals in cell lines obtained from transgenic mice that are made to express $\beta 2$-adrenergic receptor fused to RLuc ( $\beta 2$ AR-RLuc) and $\beta$ arrestin2 fused to a GFP (GFP2- $\beta$ arr2) (39). Even though this development does not count for an actual detection of endogenous proteins, it is definitely a leap in that direction. With the inception of BRETbased quantum dots (QDs) conjugates $(40,41)$, in vivo imaging in small animal models has been simplified. These new generations of BRET probes follow a similar approach as the conventional BRET systems and act as BRET acceptors for RLuc donor. Some of these QDs can emit at wavelengths as high as $800 \mathrm{~nm}$, enabling the visualization of dynamic PPIs from deep tissues of small, live animals with better resolution. 


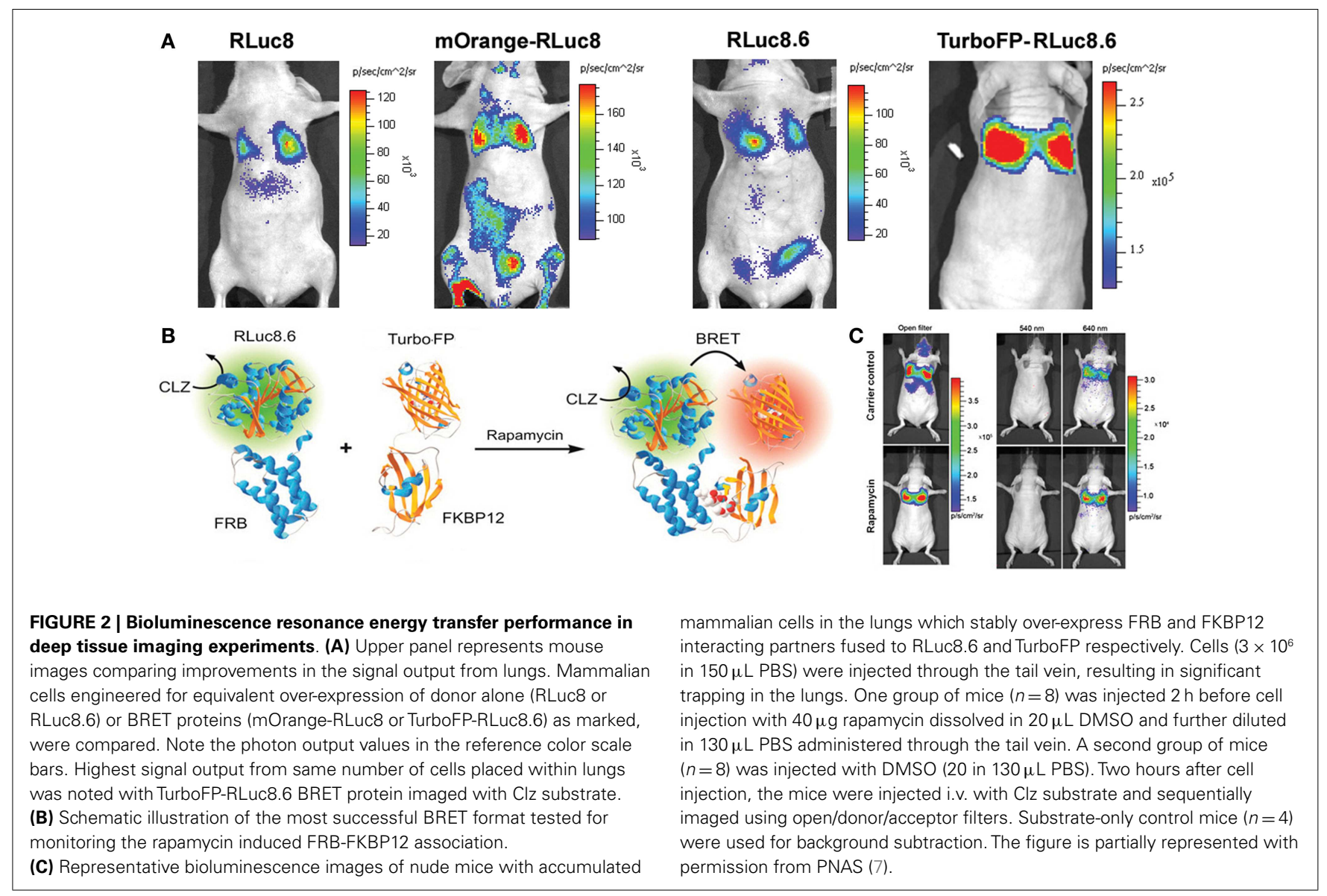

\section{CONCLUSION}

Bioluminescence-based live cell assays are becoming increasingly attractive in biological applications as they are rapid, fairly sensitive, cost effective and easy to perform, some are even acquiescent to high-throughput systems and offer several advantages in comparison to other in vitro systems. BRET has been utilized for developing diverse live cell-based assays, many of which have now been adapted in small animal research for tracking specific protein functions, phosphorylation, and protease activation events as well

\section{REFERENCES}

1. Williams NE. Immunoprecipitation procedures. Methods Cell Biol (2000) 62:449-53. doi:10.1016/ S0091-679X(08)61549-6

2. Selbach M, Mann M. Protein interaction screening by quantitative immunoprecipitation combined with knockdown (QUICK). Nat Methods (2006) 3:981-3. doi: 10.1038/nmeth972

3. Phizicky EM, Fields S. Proteinprotein interactions: methods for detection and analysis. Microbiol $\operatorname{Rev}$ (1995) 59:94-123.

4. Rediger A, Tarnow P, Bickenbach A, Schaefer M, Krude H, Gruters A, et al. Heterodimerization of hypothalamic G-protein-coupled receptors involved in weight regulation. Obes Facts (2009) 2:80-6. doi:10.1159/000209862

5. Ciruela F. Fluorescence-based methods in the study of protein-protein interactions in living cells. Curr Opin Biotechnol (2008) 19:338-43. doi:10.1016/j.copbio.2008.06.003

6. Ciruela F, Vilardaga JP, FernandezDuenas V. Lighting up multiprotein complexes: lessons from GPCR oligomerization. Trends Biotechnol (2010) 28:407-15. doi:10.1016/j. tibtech.2010.05.002

7. Dragulescu-Andrasi A, Chan CT, De A, Massoud TF, Gambhir SS. Bioluminescence resonance energy transfer (BRET)

as screening genetic and chemical modulators. By making this technology versatile, their scope for BRET-based molecular imaging of biological events from living cells and subjects will continue to expand.

\section{ACKNOWLEDGMENTS}

Research funding (BT/PR3651/MED/32/210/2011) from Department of Bioengineering, New Delhi, India to Abhijit De is acknowledged.

imaging of protein-protein interactions within deep tissues of living subjects. Proc Natl Acad Sci U S A (2011) 108:12060-5. doi:10.1073/pnas.1100923108

8. Forster T. Zwischenmolekulare Energiewanderung und Fluoreszenz. Ann Phys (1948) 2:54-75.

9. Pfleger KD, Eidne KA. Illuminating insights into protein-protein interactions using bioluminescence resonance energy transfer (BRET). Nat Methods (2006) 3:165-74. doi:10. 1038/nmeth841

10. Eidne KA, Kroeger KM, Hanyaloglu AC. Applications of novel resonance energy transfer techniques to study dynamic hormone receptor interactions in living cells.
Trends Endocrinol Metab (2002) 13:415-21. doi:10.1016/S10432760(02)00669-0

11. Xu Y, Piston DW, Johnson CH. A bioluminescence resonance energy transfer (BRET) system: application to interacting circadian clock proteins. Proc Natl Acad Sci U S A (1999) 96:151-6. doi:10.1073/pnas. 96.1.151

12. De A, Ray P, Loening AM, Gambhir SS. BRET3: a red-shifted bioluminescence resonance energy transfer (BRET)-based integrated platform for imaging protein-protein interactions from single live cells and living animals. FASEB $J$ (2009) 23:2702-9. doi:10.1096/fj.08118919 
13. De A. The new era of bioluminescence resonance energy transfer technology. Curr Pharm Biotechnol (2011) 12:558-68. doi:10.2174/ 138920111795163922

14. Bertrand L, Parent S, Caron M, Legault M, Joly E, Angers S, et al. The BRET2/arrestin assay in stable recombinant cells: a platform to screen for compounds that interact with G protein-coupled receptors (GPCRS). J Recept Signal Transduct Res (2002) 22:533-41. doi:10. 1081/RRS- 120014619

15. Dionne P, Mireille C, Labonte A, Carter-Allen K, Houle B, Joly E, et al. BRET2: efficient energy transfer from Renilla luciferase to GFP2 to measure protein-protein interactions and intracellular signaling events in live cells. In: van Dyke K, van Dyke C, Woodfork $\mathrm{K}$, editors. Luminescence Biotechnology: Instruments and Applications. Boca Raton: CRC Press (2002). p. 539-55.

16. De A, Gambhir SS. Noninvasive imaging of protein-protein interactions from live cells and living subjects using bioluminescence resonance energy transfer. FASEB J (2005) 19:2017-9.

17. De A, Loening AM, Gambhir SS. An improved bioluminescence resonance energy transfer strategy for imaging intracellular events in single cells and living subjects. Cancer Res (2007) 67:7175-83. doi:10. 1158/0008-5472.CAN-06-4623

18. Shcherbo D, Merzlyak EM, Chepurnykh TV, Fradkov AF, Ermakova GV, Solovieva EA, et al. Bright far-red fluorescent protein for whole-body imaging. Nat Methods (2007) 4:741-6. doi:10.1038/ nmeth 1083

19. Loening AM, Fenn TD, Wu AM, Gambhir SS. Consensus guided mutagenesis of Renilla luciferase yields enhanced stability and light output. Protein Eng Des Sel (2006) 19:391-400. doi:10.1093/protein/gzl023

20. Loening AM, Wu AM, Gambhir SS. Red-shifted Renilla reniformis luciferase variants for imaging in living subjects. Nat Methods (2007) 4:641-3. doi:10.1038/nmeth1070

21. Levi J, De A, Cheng Z, Gambhir SS. Bisdeoxycoelenterazine derivatives for improvement of bioluminescence resonance energy transfer assays. $J \mathrm{Am}$ Chem Soc (2007) 129:11900-1. doi:10.1021/ja073936h

22. Otto-Duessel M, Khankaldyyan V, Gonzalez-Gomez I, Jensen MC, Laug WE, Rosol M. In vivo testing of Renilla luciferase substrate analogs in an orthotopic murine model of human glioblastoma. Mol Imaging (2006) 5:57-64.

23. Chiu NH, Christopoulos TK. Two-site expression immunoassay using a firefly luciferase-coding DNA label. Clin Chem (1999) 45:1954-9.

24. Naumov P, Ozawa Y, Ohkubo K, Fukuzumi S. Structure and spectroscopy of oxyluciferin, the light emitter of the firefly bioluminescence. J Am Chem Soc (2009) 131:11590-605. doi:10.1021/ja904309q

25. Arai R, Nakagawa H, Kitayama A, Ueda H, Nagamune T. Detection of protein-protein interaction by bioluminescence resonance energy transfer from firefly luciferase to red fluorescent protein. J Biosci Bioeng (2002) 94:362-4. doi:10.1263/ jbb.94.362

26. Yamakawa Y, Ueda H, Kitayama A, Nagamune T. Rapid homogeneous immunoassay of peptides based on bioluminescence resonance energy transfer from firefly luciferase. $J$ Biosci Bioeng (2002) 93:537-42. doi: 10.1016/S1389-1723(02)80234-1

27. Koo JA, Schmidt SP, Schuster GB. Bioluminescence of the firefly: key steps in the formation of the electronically excited state for model systems. Proc Natl Acad Sci U S A (1978) 75:30-3. doi:10.1073/pnas. 75.1 .30

28. Ohmiya Y. Basic and applied aspects of color tuning of bioluminescence systems. Jpn J Appl Phys (2005) 44:6368-79. doi:10.1143/ JJAP.44.6368

29. Perroy J, Pontier S, Charest PG, Aubry M, Bouvier M. Real-time monitoring of ubiquitination in living cells by BRET. Nat Methods (2004) 1:203-8. doi:10.1038/ nmeth722

30. Carriba P, Navarro G, Ciruela F, Ferre S, Casado V, Agnati L, et al. Detection of heteromerization of more than two proteins by sequential BRET-FRET. Nat
Methods (2008) 5:727-33. doi:10. 1038/nmeth.1229

31. Navarro G, Aymerich MS, Marcellino D, Cortes A, Casado V, Mallol $\mathrm{J}$, et al. Interactions between calmodulin, adenosine A2A, and dopamine D2 receptors. J Biol Chem (2009) 284:28058-68. doi:10.1074/ jbc.M109.034231

32. Heroux M, Hogue M, Lemieux S, Bouvier M. Functional calcitonin gene-related peptide receptors are formed by the asymmetric assembly of a calcitonin receptor-like receptor homo-oligomer and a monomer of receptor activitymodifying protein-1. J Biol Chem (2007) 282:31610-20. doi:10.1074/ jbc.M701790200

33. Navarro G, Carriba P, Gandia J, Ciruela F, Casado V, Cortes A, et al. Detection of heteromers formed by cannabinoid CB1, dopamine D2, and adenosine A2A G-protein-coupled receptors by combining bimolecular fluorescence complementation and bioluminescence energy transfer. Sci World J (2008) 8:1088-97. doi:10.1100/tsw.2008.136

34. Urizar E, Yano H, Kolster R, Gales C, Lambert N, Javitch JA. CODARET reveals functional selectivity as a result of GPCR heteromerization. Nat Chem Biol (2011) 7:624-30. doi:10.1038/nchembio.623

35. Guo W, Urizar E, Kralikova M, Mobarec JC, Shi L, Filizola M, et al. Dopamine D2 receptors form higher order oligomers at physiological expression levels. EMBO $J$ (2008) 27:2293-304. doi:10.1038/ emboj.2008.153

36. Drinovec L, Kubale V, Nohr Larsen J, Vrecl M. Mathematical models for quantitative assessment of bioluminescence resonance energy transfer: application to seven transmembrane receptors oligomerization. Front Endocrinol (2012) 3:104. doi:10.3389/fendo.2012. 00104

37. Xu X, Soutto M, Xie Q, Servick S, Subramanian C, Von Arnim $\mathrm{AG}$, et al. Imaging protein interactions with bioluminescence resonance energy transfer (BRET) in plant and mammalian cells and tissues. Proc Natl Acad Sci U S A (2007) 104:10264-9. doi:10.1073/ pnas.0701987104
38. Subramanian C, Kim BH, Lyssenko $\mathrm{NN}$, Xu X, Johnson $\mathrm{CH}$, Von Arnim AG. The Arabidopsis repressor of light signaling, COP 1 , is regulated by nuclear exclusion: mutational analysis by bioluminescence resonance energy transfer. Proc Natl Acad Sci U S A (2004) 101:6798-802. doi:10.1073/ pnas.0307964101

39. Audet M, Lagace M, Silversides DW, Bouvier M. Protein-protein interactions monitored in cells from transgenic mice using bioluminescence resonance energy transfer. FASEB J (2010) 24:2829-38. doi:10. 1096/fj.09-144816

40. So MK, Loening AM, Gambhir SS, Rao J. Creating self-illuminating quantum dot conjugates. Nat Protoc (2006) 1:1160-4. doi:10.1038/ nprot.2006.162

41. Xia Z, Xing Y, So MK, Koh AL, Sinclair R, Rao J. Multiplex detection of protease activity with quantum dot nanosensors prepared by inteinmediated specific bioconjugation. Anal Chem (2008) 80:8649-55. doi: $10.1021 / \mathrm{ac} 801562 \mathrm{f}$

Conflict of Interest Statement: The authors declare that the research was conducted in the absence of any commercial or financial relationships that could be construed as a potential conflict of interest.

Received: 20 June 2013; accepted: 05 September 2013; published online: 23 September 2013

Citation: De A, Jasani A, Arora $R$ and Gambhir SS (2013) Evolution of BRET biosensors from live cell to tissue-scale in vivo imaging. Front. Endocrinol. 4:131. doi: 10.3389/fendo.2013.00131

This article was submitted to Molecular and Structural Endocrinology, a section of the journal Frontiers in Endocrinology. Copyright () 2013 De, Jasani, Arora and Gambhir. This is an open-access article distributed under the terms of the Creative Commons Attribution License (CC $B Y)$. The use, distribution or reproduction in other forums is permitted, provided the original author(s) or licensor are credited and that the original publication in this journal is cited, in accordance with accepted academic practice. No use, distribution or reproduction is permitted which does not comply with these terms. 